\title{
PARASITAS ZOONÓTICOS EM FEZES DE CÃES EM PRAÇAS PÚBLICAS DO MUNICÍPIO DE ITABUNA, BAHIA, BRASIL
}

\author{
PEDRO C. CAMPOS FILHO ${ }^{1}$, LAISE M. BARROS ${ }^{1}$, JAMILLE O. CAMPOS ${ }^{2}$, VÂNIA B. BRAGA ${ }^{1}$, IRENE M. \\ CAZORLA $^{3}$, GEORGE R. ALBUQUERQUE ${ }^{4}$, SÍLVIA M.S. CARVALHO ${ }^{5}$
}

\begin{abstract}
CAMPOS FILHO, P.C.; BARROS, L.M.; CAMPOS, J.O.; BRAGA, V.B.; CAZORLA, I.M.; ALBUQUERQUE, G. R.; CARVALHO, S.M.S. [Zoonotic parasites in dog feces at public squares in the municipality of Itabuna, Bahia, Brazil]. Parasitas zoonóticos em fezes de cães em praças públicas do município de Itabuna, Bahia, Brasil. Revista Brasileira de Parasitologia Veterinária, v. 17, n. 4, p.206-209, 2008. Departamento de Ciências Agrárias e Ambientais, Universidade Estadual de Santa Cruz (UESC), Rodovia Ilhéus-Itabuna, Km 16, Ilhéus, BA 45662-000, Brasil. E-mail: gralbu@uesc.br

The contamination of public squares by zoonotic potential parasites was evaluated at the urban areas in the municipality of Itabuna Brasil. For such, 119 fecal samples of dogs were collected at 10 public squares. After that, these feces samples were transported to the Parasitology Laboratory in the UESC and they were analyzed by Mariano and Carvalho's method. Of the total analized samples, 56.3\% show some parasitic evolutive form. Ancylostoma sp. was the most frequently (47.9\%), followed by Strongyloides stercortalis (6.7\%), Toxocara canis and Trichuris vulpis eggs (4.2\%) each, Endolimax nana cysts (2.5\%), and Giardia intestinalis and Entamoeba coli cysts (0.8\%) each.
\end{abstract}

KEY WORDS: Ascarids, Ancylostomatids, zoonoses, southestern Bahia.

\section{RESUMO}

Avaliou-se a contaminação de praças públicas da área urbana do município de Itabuna, BA, Brasil, por parasitos zoonóticos presentes em fezes de cães. Foram coletadas 119 amostras fecais de cães em 10 praças. Logo após, estas fezes foram encaminhadas ao Laboratório de Parasitologia da UESC e analisadas pelo método de Mariano e Carvalho. Do total das amostras analisadas, 56,3\% continham alguma forma evolutiva parasitária, sendo o parasita mais freqüente Ancylostoma sp. com 47,9\%, seguido por 6,7\% de Strongyloides stercortalis, 4,2\% tanto para ovos de Toxocara canis quanto de Trichuris vulpis, 2,5\% para cistos de Endolimax nana, e 0,8\% tanto para cistos de Giardia intestinalis quanto para os de Entamoeba coli.

PALAVRAS-CHAVE: Ascarídios, ancilostomídeos, zoonoses, sul da Bahia.

\footnotetext{
${ }^{1}$ Curso de Biomedicina, Universidade Estadual de Santa Cruz (UESC), Ilhéus, BA.

${ }^{2}$ Curso de Medicina Veterinária, UESC, Ilhéus, BA.

${ }^{3}$ Departamento de Ciências Exatas e Tecnológicas, UESC, Ilhéus, BA.

${ }^{4}$ Departamento de Ciências Agrárias e Ambientais, UESC, Rodovia Ilhéus-Itabuna, Km 16, Ilhéus, BA 45662-000, Brasil. Email: gralbu@uesc.br

${ }^{5}$ Departamento de Ciências Biológicas, UESC, Ilhéus, BA. Email: sissacarvalho@yahoo.com
}

\section{INTRODUÇÃO}

Animais de companhia, particularmente cães, têm um importante papel na sociedade em todo o mundo. Eles são em alguns casos companheiros importantes em muitas residências, contribuindo ao desenvolvimento físico, social e emocional de crianças e o bem-estar de seus donos, em especial idosos (JENNINGS 1997; RAINA et al., 1999).

Os cães são hospedeiros definitivos de algumas espécies de parasitos zoonóticos. O crescente número de animais domiciliados, peridomiciliados e errantes, de modo geral, em todo o Brasil, associado ao fácil acesso destes aos locais de lazer, como praças públicas e praias, têm aumentado o risco de infecção, especialmente para crianças, constituindo um problema de saúde pública (SANTAREM et al., 1998; SCAINI et al., 2003; CASTRO et al., 2005).

Dentre os parasitos observados em cães, destacam-se, os dos gêneros Toxocara, Ancylostoma, Trichuris e Giardia, alguns deles agentes da larva migrans visceral, larva migrans cutânea em humanos, e giardíase em humanos e animais (SCHANTZ, 1991; ROBERTSON et al., 2000; DUNN et al., 2002).

A contaminação de praças públicas por parasitos procedentes de cães tem sido alvo de pesquisas tanto no Brasil quanto em outros países, e todas demonstram a importância das 
praças como ambiente propício à transmissão de agentes patogênicos, inclusive parasitários (CORREA; MOREIRA, 1995, 1996; SANTARÉM et al., 1998; ARAUJO et al., 1999; MERCADO et al., 2004; CAPUANO; ROCHA 2006).

O presente trabalho foi realizado com o objetivo de avaliar a ocorrência de parasitos zoonóticos em fezes de cães oriundas de praças públicas da área urbana do município de Itabuna, Bahia, Brasil.

\section{MATERIAL E MÉTODOS}

Foram coletadas, de Maio de 2004 a Maio de 2005, 119 amostras fecais de cães, com aspecto de não ressecadas, em dez praças públicas da área urbana (Figura 1) do município de Itabuna (Latitude 1447’08" Sul; Longitude 39¹6’49" Oeste); sendo estas divididas em duas áreas, para melhor qualificar a análise dos dados observados. Essa divisão levou em conta à geografia da cidade de Itabuna, que é cortada pelo Rio Cachoeira, ficando uma área com seis praças e a outra com quatro. A quantidade de amostras utilizadas neste estudo foi decorrente do número de fezes existentes nas praças nos dias de coleta. Estas praças foram escolhidas por serem bastante freqüentadas pela população local.

As amostras fecais foram coletadas a cada quinze dias, com o auxílio de uma espátula, e armazenadas em coletores de fezes estéreis, previamente identificados, acondicionados em caixa isotérmica com gelo reciclável e encaminhadas, no mesmo dia, ao Laboratório de Parasitologia Médica da Universidade Estadual de Santa Cruz e processadas através do método de Mariano e Carvalho (MARIANO et al., 2005). A análise foi realizada à microscopia óptica em objetivas de 10X e 40X.

O teste Qui-quadrado foi utilizado para avaliar a associação entre a ocorrência de parasitos e a área de localização geográfica das praças das quais foram coletadas as fezes. Foram considerados estatisticamente significantes os valores de $\mathrm{p}<0,05$.

\section{RESULTADOS E DISCUSSÃO}

Das 119 amostras coletadas, 67 (56,3\%) foram positivas. Destas, 54 (45,4\%) tinham apenas um tipo de espécie parasitária, enquanto que $13(10,9 \%)$ estavam poliparasitadas. A ocorrência dos diferentes parasitos está apresentada na Tabela 1 . Das 57 (47,9\%) amostras que foram positivas para Ancylostoma sp., em 13,4\% (16/119) foram encontradas larvas rabditóides ou filarióides. Vale considerar que o encontro de larvas filárióides indica que o ambiente oferece condições necessárias ao desenvolvimento desse tipo de parasita, possibilitando a ocorrência de larva migrans cutânea (FERREIRA et al., 2003). Este também foi o parasito mais recuperado nos estudos de Araújo et al. (1999); Scaini et al. (2003); Castro et al., (2005); e Capuano e Rocha (2006), apresentando uma

Tabela 1. Diagnóstico de parasitos em fezes de cães em praças públicas da cidade de Itabuna, BA, no período de maio de 2004 a maio de 2005.

\begin{tabular}{|c|c|c|c|}
\hline \multicolumn{2}{|l|}{ Diagnóstico } & \multicolumn{2}{|c|}{ Amostra $^{a}$} \\
\hline Parasita & Forma & Positivos & Ocorrência (\%) \\
\hline Ancylostoma sp. & Ovo/Larva & 57 & 47,9 \\
\hline Strongyloides stercoralis & Ovo & 08 & 6,7 \\
\hline Toxocara canis & Ovo & 05 & 4,2 \\
\hline Trichuris vulpis & Ovo & 05 & 4,2 \\
\hline Ameba $^{b}$ & Cisto & 04 & 3,7 \\
\hline Giardia & Cisto & 01 & 0,8 \\
\hline
\end{tabular}

a 119 amostras analisadas

${ }^{\mathrm{b}}$ Entomoeba coli, Endolimax nana

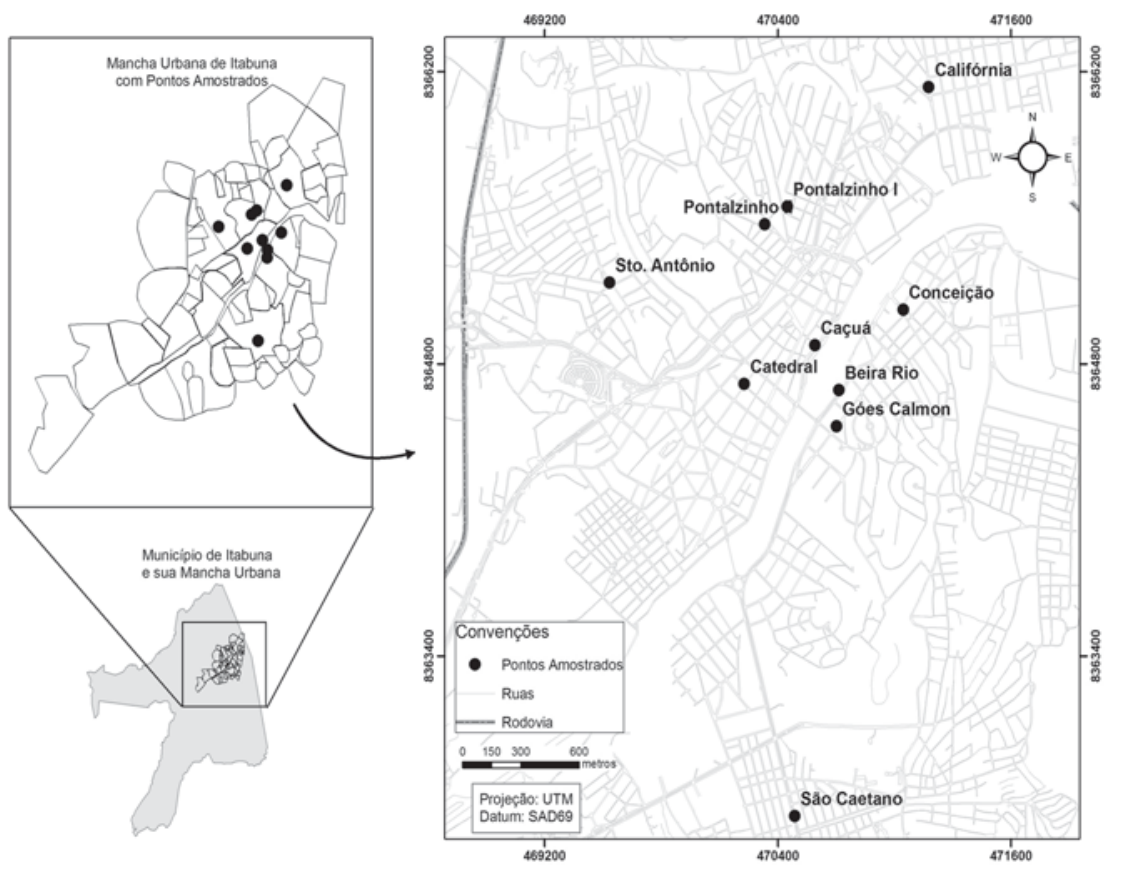

Figura 1. Mapa de localização das praças públicas estudadas no município de Itabuna, BA. 
positividade entre $41,7 \%$ e $71,3 \%$. Todos também enfocaram a contaminação de áreas públicas por fezes caninas.

O contato com solo contaminado por fezes de cães ou com as próprias fezes contendo o parasito pode ser responsável pelo aparecimento de casos de larva migrans cutânea . Isso é particularmente comum em áreas com alta umidade, especialmente entre banhistas que passeiam em areia molhada contaminada por larvas deste parasito (SCHANTZ, 1994). Ancylostoma caninum tem sido apontado como causador de enterite eosinofílica em humanos, principalmente em países tropicais (WALKER et al., 1995). O município de Itabuna fica situado na região sul do estado da Bahia, uma área com clima quente e úmido, em virtude da presença da mata Atlântica, preservada pelas plantações de cacau, o que torna o local propício para a viabilidade e disseminação deste parasito.

O segundo parasita mais freqüente com $6,7 \%$ foi Strongyloides stercoralis, resultado este superior ao observado por Scaini et al. (2003) no Balneário Cassino, RS, com positividade de 3,0\% (7/237). Em seres humanos $S$. stercoralis raramente causa alterações clínicas, mas quando apresenta, geralmente está associado a manifestações gastro-intestinais, como dores abdominais, náusea e vômito (RODRIGUES et al., 2001).

$\mathrm{O}$ terceiro e o quarto helmintos mais observados foram ovos de T. canis e T. vulpis, com 4,2\%, cada um. Porcentagem superior foi registrada à recuperação de $T$. vulpis das fezes de cães em logradouros públicos no Balneário Cassino, RS, e em Ribeirão Preto, SP, com 32,5\% e 15,7\%, respectivamente (SCAINI et al., 2003; CAPUANO; ROCHA 2006). Ademais, vale ressaltar que $T$. vulpis pode desencadear em humanos dor abdominal, náusea e vômito (DUNN et al. 2002), além de já ter sido responsabilizado como responsável por larva migrans visceral (MASUDA et al., 1987).

A notificação de ovos de $T$. canis, em amostras fecais ou no solo de áreas públicas, tem sido comum no Brasil, com percentuais variando de 1,0\% a 100,0\% (CHIEFFI; MÜLLER, 1976; ALCÂNTARA et al., 1989; CORREA; MOREIRA, 1995, 1996; SANTARÉM et al., 1998; ARAÚJO et al. 1999; SCAINI et al., 2003; FERREIRA et al., 2003; MENTZ et al., 2004; CASTRO et al., 2005; CAPUANO; ROCHA, 2006).

Quanto aos cistos encontrados, foi observada a presença de três $(2,5 \%)$ amostras contaminadas com cistos de Endolimax nana e uma (0,8\%) com Entamoeba coli. Embora considerados parasitos apatogênicos, o diagnóstico destas espécies é importante pela dificuldade em diferenciar esses comensais, colonizadores dos intestinos do homem e de animais, com o de E. histolitica, ameba de importância médico veterinária. Obteve-se apenas uma amostra positiva para cistos de Giardia sp. (0,8\%), esse resultado foi abaixo do encontrado por Capuano e Rocha (2006), que obtiveram 10,2\% em fezes coletadas de áreas públicas no município de Ribeirão Preto, SP.

As divergências numéricas encontradas entre os estudos apontados podem estar associado a uma série de fatores, como número de cães que circulam nas praças, a diferença climática entre as regiões estudadas, diferentes metodologias em-
Tabela 2. Associação entre a presença de parasitas em fezes de cães e as praças públicas, agrupadas em duas áreas, no município de Itabuna, BA.

\begin{tabular}{|c|c|c|c|c|}
\hline \multirow[t]{2}{*}{ Área } & \multicolumn{2}{|c|}{ Amostras } & \multirow[t]{2}{*}{$\chi^{2}$} & \multirow[t]{2}{*}{ Valor de $p$} \\
\hline & Positivas & Negativas & & \\
\hline$U^{a}{ }^{a}$ & 34 & 17 & 3,19 & 0,074 \\
\hline Dois $^{b}$ & 33 & 35 & & \\
\hline
\end{tabular}

a Praças Califórnia, Pontalzinho 1 e 2, Santo Antonio, Caçuá, Catedral

b Praças Conceição, Góes Calmon, Beira Rio, São Caetano

pregadas, entre outras. Neste trabalho, o método de Mariano e Carvalho (MARIANO et al., 2005) se mostrou eficiente, pois foi capaz de sedimentar facilmente os ovos, cistos e, principalmente, as larvas dos parasitos.

O teste Qui-quadrado não apontou diferença significativa entre as proporções de amostras parasitadas nas duas áreas estudadas (Tabela 2). Araújo et al. (1999) também agrupou as praças pelas regiões do município e não obteve diferenças significativas. De todo modo, é relevante ressaltar que as praças do Rio Cachoeira (Beira Rio) e São Caetano, maiores, com grande fluxo de pessoas e animais, foram as mais contaminadas, contendo 31 e 22 amostras fecais, respectivamente. Por sua vez, na Praça Góes Calmon, bairro de classe alta, foi coletada apenas uma amostra fecal, sendo obviamente, a menos contaminada.

\section{CONCLUSÕES}

Os resultados demonstraram a presença de ovos, larvas e cistos de parasitos zoonóticos em fezes de cães oriundas de praças públicas no município de Itabuna, indicando esses locais como fonte em potencial na transmissão de parasitos zoonóticos para seres humanos.

Agradecimentos: Maurício Santana Moreau pela confecção do mapa de localização das praças públicas na cidade de Itabuna.

\section{REFERÊNCIAS BIBLIOGRÁFICAS}

ALCÂNTARA, N.; BAVIA, E.; SILVÃO, R.M. Environmental contamination by Toxocara spp. eggs in public areas of Salvador, Bahia State, Brasil. Revista da Sociedade Brasileira de Medicina Tropical, v. 22, n. 4 , p. 187-190, 1989.

ARAÚJO, F.R.; CROCCI, A.J.; RODRIGUES, R.G.C.; AVALHAES, J.S.; MIYOSHI, M.I.; SALGADO, F.P.; SILVA, M.A.; PEREIRA, M.L. Contaminação de praças públicas de Campo Grande, Mato Grosso do Sul, Brasil, por ovos de Toxocara e Ancylostoma em fezes de cães. Revista da Sociedade Brasileira de Medicina Tropical, v. 32, n. 5, p. 581-583, 1999.

CAPUANO, D.M.; ROCHA, G.M. DE. Ocorrência de parasitas com potencial zoonótico em fezes de cães coletadas em áreas públicas do município de Ribeirão Preto, SP, Brasil. Revista Brasileira de Epidemiologia, v. 9, n. 1, p. 8186, 2006. 
CASTRO, J.M.; SANTOS, S.V.; MONTEIRO, N.A. Contaminação de canteiros da orla marítima do município de Praia Grande, São Paulo, por ovos de Ancylostoma e Toxocara em fezes de cães. Revista da Sociedade Brasileira de Medicina Tropical, v. 38, n. 2, p. 199-201, 2005.

CORRÊA, G.L.B.; MOREIRA, W.S. Soil contamination due to eggs of Ancylostoma spp. in public parks of the city of Santa Maria, RS, Brazil. Revista da Faculdade de Zootecnia Veterinária e Agronomia, v. 2-3, n. 1, p. 15-17, 1995/1996.

CHIEFFI, P.P.; MÜLLER, E.E. Prevalência de parasitismo por Toxocara canis em cães e presença de ovos de Toxocara sp. no solo de localidades públicas da zona urbana do município de Londrina, Estado Paraná, Brasil. Revista de Saúde Pública, v. 10, n. 4, p. 367-372, 1976.

DUNN, J.J.; COLUMBUS, S.T.; ALDEEN, W.E.; DAVIS, M.; CARROLL, K.C. Trichuris vulpis recovered from a patient with chronic diarrhea and five dogs. Journal of Clinical Microbiology, v. 40, n. 7, p. 2703-2704, 2002.

FERREIRA, C.; MACHADO, S.; SELORES, M. Larva Migrans Cutânea em idade pediátrica: a propósito de um caso clínico. Revista Nascer e Crescer, v. 12, n. 4, p. 261264, 2003.

JENNINGS, L.B. Potencial benefits of pet ownership in health promotion. Journal of Holistic Nursing, v. 15, n. 4, p. 358372, 1997.

MARIANO, M.L.M.; CARVALHO, S.M.S.; MARIANO, A.P.M.; ASSUNÇÃO, F.R. DE; CAZORLA, I.M. Uma nova opção de diagnóstico parasitológico: Método de Mariano e Carvalho. Newslab, v. 68, p. 132-140, 2005.

MASUDA, Y.; KISHIMOTO, T.; ITO, H.; TSUJI, M. Visceral larva migrans caused by Trichuris vulpis presenting as a pulmonary mass. Thorax, v. 42, n. 12, p. 990-991, 1987.

MERCADO, R.; UETA, M.T.; CASTILLO, D.; MUÑOZ, V.; SCHENONE, H. Exposure to larva migrans syndromes in squares and public parks of cities in Chile. Revista de Saúde Pública, v. 38, n. 5, p. 729-731, 2004.

MENTZ, M.B.; ROTT, M.B.; JACOBSEN, S.I.V.; BALDO,
G.; RODRIGUES-JUNIOR, V. Frequency of Toxocara spp. in three public parks of Porto Alegre city - Brazil. Revista de Patologia Tropical, v. 33, n. 1, p. 105-112, 2004.

NUNES, C.M.; PENA, F.C.; NEGRELLI, G.B.; ANJO, C.G.S.; NAKANO, M.M.; STOBBE, N.S. Ocorrência de larva migrans na areia de areias de lazer das escolas municipais de ensino infantil, Araçatuba, SP, Brasil. Revista de Saúde Pública, v. 34, n. 6, p. 656-658, 2000.

RAINA, P.; WALTNER-TOEWS, D.; BONNET, B.; WOODWARD, C.; ABERNATHY, T. Influence of companion animals on the physical and psychological health of older people: an analysis of a one-year longitudinal study. Journal of the American Geriatrics Society, v. 47, n. 3, p. 323-329, 1999.

ROBERTSON, I.D.; IRWIN, P.J.; LYMBERY, A.J.; THOMPSON, R.C.A. The role of companion animals in the emergence of parasitic zoonoses. International Journal of Parasitology, v. 30, n. 12-13, p. 1369-1377, 2000.

RODRIGUES, M.A.M.; FRÓES, R.C.; ANEFALOS, A.; KOBAYASI, K. Invasive enteritis by Strongyloides stercoralis presenting as acute abdominal distress under corticosteroid therapy. Revista do Hospital das Clínicas Faculdade de Medicina de São Paulo, v. 56, n. 4, p. 103106, 2001.

SANTAREM, V.A.; SERTOR, I.F.; BERGAMO, F.M.M. Contamination by Toxocara spp.s eggs in public parks and squares in Botucatu, São Paulo State, Brazil. Revista da Sociedade Brasileira de Medicina Tropical, v. 31, n. 6, p. 529-532, 1998.

SCAINI, C.J.; TOLEDO, R.N.; LOVATEL, R.; DIONELLO, M.A.; GATTI, F.A.; SUSIN, L.; SIGNORINI, V.R.M. Environment contamination by helminth eggs and larvae in dog feces from central area of Cassino Beach, Rio Grande do Sul. Revista da Sociedade Brasileira de Medicina Tropical, v. 36, n. 5, p. 617-619, 2003.

SCHANTZ, P.M. Parasitic zoonoses in perspective. International Journal of Parasitology, v. 21, n. 2, p. 161-170, 1991.

Recebido em 24 de maio de 2007.

Aceito para publicação em 29 de dezembro de 2008. 